\title{
RESISTÊNCIA DE SOJA A INSETOS: VIII. IAC 78-2318, LINHAGEM COM RESISTÉNCIA MÚLTIPLA (1)
}

\author{
ANDRÉ LUR LOURENÇĀO $\left({ }^{2,4}\right)$ e MANOEL ALBINO COELHO DE MIRANDA $\left({ }^{3,4}\right.$ )
}

\begin{abstract}
RESUMO
Estudou-se, em comparação com outros genótipos de soja, o comportamento da linhagem IAC 78-2318, em relação à oviposiçāo e colonização da mosca-branca Bemisia tabaci (Genn.) e à área foliar consumida por besouros crisomelideos e lagartas. Em Campinas, SP, em 1981, em casa de vegetação, submeteram-se os cultivares Santa Rosa, Paraná, BR-1, Bossier, IAC 8 e IAC 12 e as linhagens IAC 73-228, IAC 78-2318, D72-9601-1, PI 171451, PI 229358 e PI 274454 à infestação artificial de adultos da mosca--branca. IAC 78-2318, embora apresentando alto número de ovos, teve colonização baixa, próxima aos materiais mais resistentes ( $P$ I 171451 e PI 229358). Em Santo Antonio de Posse, SP, em 1985, em campo, IAC 78-2318, quando comparado com IAC 80-596-2, 'Santa Rosa', 'IAC 8' e 'IAC 11', mostroù a menor perda de área foliar devida à alimentação de coleópteros crisomelídeos, principalmente Cerotoma arcuata (Oliv.) e Diphaulaca viridipennis Clark, e de lagartas, com predominância de Anticarsia gemmatalis (Hubn.). Como já havia sido registrado anteriormente baixo dano de Epinotia aporema (Wals.) e de percevejos pentatomídeos em IAC 78-2318, com as observaçōes presentes essa linhagem fica caracterizada como portadora de resistência múltipla a insetos.
\end{abstract}

Termos de indexação: soja, Glycine max (L) Merrill; mosca-branca, Bemisia tabaci (Genn.); Chrysomelidae; insetos desfolhadores; resistência mútipla a insetos.

( $\left.{ }^{1}\right)$ Trabalho apresentado ao X Congresso Brasileiro de Entomologia, realizado no Rio de Janeiro (RJ), de 26 a 31 de janeiro de 1986. Recebido para publicação em 22 de julho de 1986. nas (SP).

${ }^{(2)}$ Seção de Entomologia Fitotécnica, Instituto Agronômico (IAC), Caixa Postal 28, 13001 Campi-

( $\left.{ }^{3}\right)$ Seção de Leguminosas, IAC.

(4) Bolsista do CNPq. 


\section{INTRODUÇÃO}

Pouco tempo após a descoberta de que a introdução PI 229358 possui resistência múltipla a insetos (DUYN et al., 1971; CLARK et al., 1972; HATCHETT et al., 1976; KILEN et al., 1977), já haviam sido obtidas linhagens descendentes desse material com resistência também a várias espécies de insetos (HATCHETT et al., 1979; TURNIPSEED \& KOGAN, 1976).

LAMBERT \& KILEN (1984), estudando o comportamento da PI 229358 , de duas linhagens descendentes dessa introdução e de três cultivares em relação a quatro lagartas, obtiveram evidências de que, quando a PI 229358 é usada como fonte de resistência, a seleção para resistência a uma das espécies pode resultar em seleção indireta para resistência às outras.

No Brasil, certas linhagens, cuja genealogia apresenta a PI 229358, foram observadas em relação ao ataque de insetos com resultados positivos. D72-9601-1 exibiu baixo desfolhamento devido à alimentação de lagartas em condiçōes de campo (REZENDE et al., 1980); IAC 78-2318 e IAC 78-3278 foram as menos atacadas em seus ponteiros pela broca das axilas Epinotia aporema (Wals.) (LOURENÇÃO \& MIRANDA, 1983). Com respeito a danos causados por percevejos pentatomideos, a linhagem IAC 78-2318 comportou-se como resistente em experimentação conduzida durante dois anos agrícolas consecutivos (1980/81 e 1981/82) por LOURENÇĀO et al. (1987) e em 1983/84 e 1984/85 por ROSSETTO et al. (1986).

Como a linhagem IAC 78-2318 apresenta resistência aos percevejos pentatomideos, que constituem o principal grupo de pragas da soja para as condiçōes do Estado de São Paulo, e também à broca E. aporema, é de grande interesse saber seu comportamento em relação a outros insetos que se alimentam dessa leguminosa. Assim, estudou-se o seu comportamento em relação à oviposição e colonização da mosca-branca Bemisia tabaci (Genn.) e ao dano foliar causado por insetos desfolhadores (besouros crisomelídeos e lagartas).

\section{MATERIAL E MÉTODOS}

\subsection{Oviposição e colonização de Bemisia tabaci}

Utilizaram-se os cultivares Santa Rosa, Paraná, BR-1, Bossier, IAC 8 e IAC 12 mais as linhagens IAC 73-228, IAC 78-2318, D72-9601-1, PI 171451, PI 229358 e PI 274454. Em 5-6-81, em casa de vegetação, sementes desses materiais foram tratadas com inoculante e semeadas em vasos de aluminio com capacidade para 1,5 litro; logo após a germinação, mediante desbaste, deixaram-se duas plantas por vaso. Os vasos foram dispostos segundo um delineamento de blocos ao acaso, com dez repetiçóes, sendo cada parcela formada por um vaso 
com duas plantas. Quando as plantas emitiram o par de folhas unifoliadas, procedeu-se à infestação da casa de vegetação com adultos de moscas-brancas, presentes em grande número em plantas de tomate envasadas, as quais foram colocadas entre os blocos. Cinco dias depois, coletaram-se as duas folhas unifoliadas das plantas, num total de quatro folhas por parcela, para contagem do número de ovos. As folhas foram passadas em medidor de área foliar, obtendo-se o número de ovos/centimetro quadrado. A seguir, procedeu-se a novo desbaste, deixando-se apenas uma planta por parcela. As plantas permaneceram nesse ambiente até a fase de maturação das vagens, época em que se avaliou a colonizaçăo da mosca-branca, considerando-se a planta toda e utilizando-se a seguinte escala de notas: 0: ausência de ninfas + exúvias; 1 : número reduzido de ninfas + exúvias; 2: número médio; e 3: número alto (Figura 1).

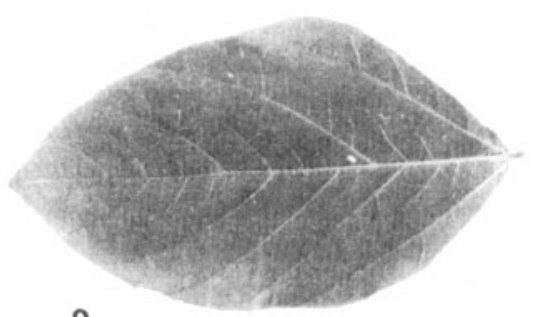

0

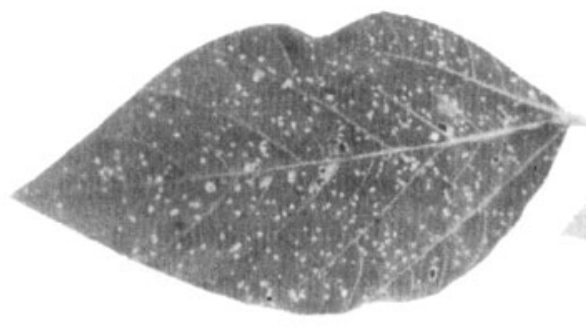

2

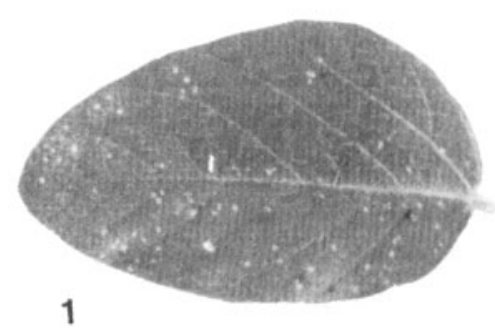

1

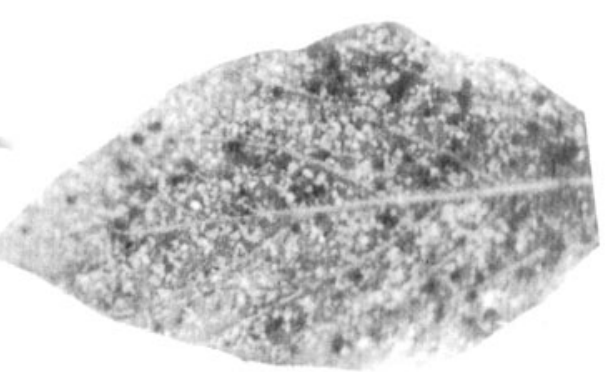

3

Figura 1. Escala de notas usada para estimar a colonização de Bemisia tabaci em soja, em casa de vegetação. Campinas, 1981. 
Os dados referentes ao nümero de ovos e às notas foram convertidos, respectivamente, em $\log x+1$ e $\sqrt{x+2}$ e, as médias, comparadas pelo teste de Tukey, ao nivel de $5 \%$.

\subsection{Avaliação de área foliar consumida por crisomelídeos e lagartas}

Em 16/11/84, instalou-se no municipio de Santo Antonio de Posse, SP, um experimento em campo a fim de estudar o comportamento de três cultivares (Santa Rosa, IAC 8 e IAC 11) e duas linhagens (IAC 78-2318 e IAC 80-596-2) de soja diante do ataque de insetos. O delineamento usado foi o de blocos ao acaso, com os cinco tratamentos repetidos oito vezes. Cada parcela se constituiu de vinte linhas espaçadas $0,60 \mathrm{~m}$ entre si e com $25 \mathrm{~m}$ de comprimento. No pleno florescimento do material, com exceção de IAC 11, que iniciava esse estádio, efetuou-se uma avaliação de dano, estimando-se visualmente o porcentual foliar perdido pela alimentação dos insetos desfolhadores em cada parcela. Os valores foram transformados em $\log x$ e, as médias, comparadas pelo teste de Tukey, ao nivel de $5 \%$.

\section{RESULTADOS E DISCUSSÃO}

Os dados médios de oviposição e colonização de Bemisia tabaci acham-se no quadro 1 . Observa-se que a PI 274454 apresenta alta suscetibilidade à postura e à colonização da mosca-branca, embora seja resistente à lagarta-enroladeira Hedilepta indicata (Fabr.) (LOURENÇĀO et al., 1985) e a percevejos pentatomideos (ROSSETTO et al., 1986). 'Santa Rosa' mostrou o mais alto grau de colonização, indicando ser excelente planta hospedeira e podendo ser usada para criação do inseto para fins de pesquisa. 'IAC 12' e 'Paraná' também se situaram entre os mais colonizados, embora o último exibisse baixo número de ovos. ROSSETTO et al. (1977), estudando a oviposição de B. tabaci em seis cultivares e três introduçōes de soja, em três ensaios com idades diferentes de plantas, registraram também um pequeno número de ovos colocados sobre as folhas de 'Paranå' em comparação com os materiais resistentes, PI 229358 e PI 171451, no ensaio das plantas mais novas. Os cultivares BR-1 e Bossier, citados por LINK et al. (1979) como os menos infestados pela mosca-branca em experimento de campo com 26 cultivares, apresentaram neste trabalho, a exemplo de D72-9601-1 e IAC 78-2318, baixa colonização. O comportamento dessas duas linhagens pode ser explicado por ambas terem sido originadas a partir de cruzamento envolvendo a PI 229358, resistente ao inseto, mesmo não tendo sido feita seleção para esse fim. As introduçōes PI 171451 e PI 229358 sofreram oviposição significativamente inferior, confirmando trabalhos de ROSSETTO et al. (1977) e LOURENÇĀO (1980), e também tiveram a menor colonização. 
QUADRO 1. Oviposição e colonização de Bemisia tabaci em genótipos de soja, em casa de vegetação. Campinas, 1981

\begin{tabular}{|c|c|c|c|}
\hline $\begin{array}{l}\text { Cultivares } \\
\text { e linhagens }\end{array}$ & Origem & Ovos $/ \mathrm{cm}^{2}$ & Colonizaçāo $\left(^{1}\right)$ \\
\hline 'Santa Rosa' & D49-772 $\times$ La 41-1219 & $18,8 \mathrm{ab}\left({ }^{2}\right)$ & $2,9 a\left({ }^{2}\right)$ \\
\hline PI 274454 & Okinawa, Japāo & $26,7 a$ & $2,6 a b$ \\
\hline 'IAC 12’ & 'Paraná' x IAC 73-231 & $12,5 a b$ & $2,5 \mathrm{abc}$ \\
\hline 'Paranå' & 'Hill' x D52-810 & $9,7 \mathrm{~b}$ & $2,4 a b c d$ \\
\hline IAC 73-228 & 'Hill' x PI 274454 & $16,4 a b$ & 1,9abcde \\
\hline 'IAC 8' & 'Bragg' x E70-51 & $12,3 a b$ & $1,8 \mathrm{bcdef}$ \\
\hline 'Bossier' & Seleção dentro de 'Lee' & $16,9 \mathrm{ab}$ & 1,2efg \\
\hline 'BR-1' & "Hill' x L 356 & $15,3 a b$ & $1,2 \mathrm{efg}$ \\
\hline IAC 78-2318 & D72-96-1 x IAC 73-227 & $16,1 a b$ & 1, eefg \\
\hline D72-9601-1 & D66-8666 x ('Bragg' x PI 229358) & $9,2 \mathrm{~b}$ & $1,0 \mathrm{efg}$ \\
\hline PI 171451 & Japäo & $10,1 b$ & $0,8 \mathrm{fg}$ \\
\hline PI 229358 & Tóquio, Japão & $9,8 b$ & $0,7 \mathrm{~g}$ \\
\hline C.V. $(\%)$ & & 24,0 & 9,6 \\
\hline
\end{tabular}

(1) 0: sem colonizaçăo; 1: baixa; ₹: média; 3: alta colonizaçăo.

(2) Médias seguidas da mesma letra na coluna não diferem significativarnente entre si pelo teste de Tukey a $5 \%$.

$\mathrm{Na}$ avaliaçāo de área foliar consumida por crisomelldeos e lagartas, durante o estádio vegetativo e início do florescimento, verificourse no campo a presença principalmente de crisomelideos, com prédominância de Cerotoma arcuata (Oliv.) e Diphaulaca viridipennis Clark; as lagartas, em sua maiơria Anticarsia gemmatalis (Hubn.), apareceram ao final do estádio vegetativo e inicio do florescimento, representando pequena parcela entre os desfolhadores.

Os resultadós obtidos - Quadro 2 - evidenciam o bom comportamento da linhagem IAC 78-2318, que apresentou o menor desfolhamento devido à alimentação dos besouros e lagartas, enquanto 'IAC 8' e 'Santa Rosa' exibiram as mais altas perdas foliares. $E$ interessante mencionar que, em ensaios preliminares conduzidos pela Seção de Leguminosas no Centro Experimental de Campinas, em vários anos, sempre foi observado menor desfolhamento em IAC 78-2318 em comparação com outros materiais, pela ação de insetos mastigadores. 
QUADRO 2. Desfolhamento médio causado por coleópteros crisomelídeos e lagartas em genótipos de soja, em campo. Santo Antonio de Posse, 1985

\begin{tabular}{llc}
\hline $\begin{array}{c}\text { Cultivares } \\
\text { e linhagens }\end{array}$ & \multicolumn{1}{c}{ Origem } & $\begin{array}{c}\text { Área foliar } \\
\text { comida (') }\end{array}$ \\
\hline & & $\%$ \\
'IAC 8' & 'Bragg' x E 70-51 & $18 \mathrm{a}$ \\
'Santa Rosa' & D49-772 x La 41-1219 & $15 \mathrm{a}$ \\
IAC 80-596 & IAC 73-228 x 'UFV-1' & $10 \mathrm{~b}$ \\
'IAC 11' & 'Paranå' x ('Davis' x IAC 73-1364) & $9 \mathrm{bc}$ \\
IAC 78-2318 & D72-9601 x IAC 73-227 & $5 \mathrm{c}$ \\
\hline C.V. (\%) & & 12,3 \\
\hline
\end{tabular}

(1) Médias seguidas da mesma letra não diferem significativamente entre si pelo teste de Tukey a $5 \%$.

Em experimento durante dois anos consecutivos, verificou-se que a linhagem IAC 78-2318 foi pouco danificada pela broca E. aporema (LOURENÇĀO \& MIRANDA , 1983) e por percevejos pentatomideos (ROSSETTO et al., 1986; LOURENÇĀO et al., 1987). Em face dos resultados de comportamento obtidos em presença da mosca-branca $B$. tabaci e de insetos desfolhadores, evidencia-se nesse material a presença de resistência múltipla a insetos. Esta caracteristica poderá ser aproveitada em programa de melhoramento de soja para resistência a insetos, visto ser IAC 78-2318 um material já melhorado, com alguns fatores negativos (porte baixo, baixa produtividade), mas que poderão ser eliminados mediante seleçōes nas progênies resultantes.

\section{SUMMARY}

\section{RESISTANCE OF SOYBEAN TO INSECTS. VIII. IAC 78-2318 LINE WITH MULTIPLE INSECT RESISTANCE}

The performance of the soybean line IAC 78-2318 in relation to oviposition and colonization by the whitefly Bemisia tabaci (Genn.) and to defoliation by caterpillars and chrysomelidae was studied in comparison to other varieties. At Campinas, State of Sāo Paulo - Brazil, in greenhouse, the cultivars Santa Rosa, Parana, BR-1, Bossier, IAC 8 and IAC 12, and the lines IAC 73-228, IAC 78-2318, D72-9601-1, PI 171451, PI 229358 e PI 274454 were submitted to artificial infestation of whitefly adults from tomato plants highly infested. Despite the high number of eggs in the IAC 78-2318 
folioles, this line had a low colonization, comparable to the more resistants lines (PI 171451 and PI 229358). At Santo Antonio de Posse, SP - Brazil, in the field, when compared with 'IAC 11', 'IAC 8', 'Santa Rosa' and IAC 80-596-2 , the IAC 78-2318 showed the lowest defoliation due to the attack of chrysomelidae, mainly Cerotoma arcuata (Oliv.) and Diphaulaca viridipennis Clark, and caterpillars, mainly Anticarsia gemmatalis (Hubn.). The IAC 78-2318 has been cited as a line with low damage by Epinotia aporema (Wals.) and by stink bugs; so, with the present results, it was characterized the presence of multiple insect resistance in this line.

Index terms: soybean; Glycine max (L.) Merril;; whitefly, Bemisia tabaci (Genn.); Chrysomelidae; defoliators; multiple insect resistance; plant resistance.

\section{REFERÊNCIAS BIBLIOGRÁFICAS}

CLARK, W.J.; HARRIS, F.A.; MAXWELL, F.G. \& HARTWIG, E.E. Resistance of certain soybean cultivars to bean leaf beetle, striped blister beetle and bollworm. Journal of Economic Entomology, 65(6):1669-1672, 1972.

DUYN, J.W. van; TURNIPSEED, S.G. \& MAXWELL, J.D. Resistance in soybeans to the Mexican bean beetle. I. Sources of resistance. Crop Science, 11:572-573, 1971.

HATCHETT, J.H.; BELAND, G.L. \& HARTWIG, E.E. Leaf-feeding resistance to bollworn and tobacco budworn in three soybean plant introductions. Crop Science, 16:277-280, 1976.

; - \& KILEN, T.C. Identification of multiple insect resitant soybean lines. Crop Science, 19:557-559, 1979.

KILEN, T.C.; HATCHETT, J.H. \& HARTWIG, E.E. Evaluation of early generation soybeans for resistance to soybean looper. Crop Science, 17:397-398, 1977.

LAMBERT, L. \& KILEN, T.C. Multiple insect resistance in several soybean genotypes. Crop Science, 24:887-890, 1984.

LINK, D.; COSTA, E.C.; CARVALHO, D.; TARRAGÓ, M.F.S. \& VEIGA, P. Infestação da mosca-branca Bemisia tabaci (Gennadius) em soja. In: SEMINÁRIO NACIONAL DE PESQUISA DE SOJA, 1., Londrina, 1978. Londrina, EMBRAPA/Centro Nacional de Pesquisa da Soja, 1979. v.2, p.23-27.

LOURENÇÃO, A.L. Bemisia tabaci (Gennadius, 1889) (Homoptera:Aleyrodidae) em soja (Glycine max (L.) Merrill): influência da variedade, da idade da planta e de cruzamentos intervarietais sobre a oviposiçāo e desenvolvimento do inseto. Piracicaba, ESALQ/USP, 1980. 58p. Dissertação (Mestrado)

\& MIRANDA, M.A.C. de. Resistência de soja a insetos. I. Comportamento de linhagens e cultivares em relação a Epinotia aporema (Wals.) (Lepidoptera:Tortricidae). Bragantia, Campinas, 42:203-209, 1983.

\& NAGAI, V. Resistência de soja a insetos: VII. Avaliação de danos de percevejos em cultivares e linhagens. Bragantia, Campinas, 46(1):45-57, 1987. 
LOURENÇÃO, A.L; ROSSETTO, C.J. \& MIRANDA, M.A.C. de. Resistência de soja a insetos. IV. Comportamento de cultivares e linhagens em relação a Hedilepta indicata (Fabr.). Bragantia, Campinas, 44(1):149-157, 1985.

REZENDE, J.A.M.; MIRANDA, M.A.C. de. \& MASCARENHAS, H.A.A. Comportamento de cultivares de soja em relação à área foliar comida por lagartas das folhas. Bragantia, Campinas, 39:161-165, 1980.

ROSSETTO, C.J.; IGUE, T.; MIRANDA, M.A.C. de \& LOURENÇÃO, A.L. Resistência de soja a insetos: Vl. Comportamento de genótipos em relaçāo a percevejos. Bragantia, Campinas, 45(2):323-335, 1986.

ROSSETTO, D.; COSTA, A.S.; MIRANDA, M.A.C. de; NAGAl, V. \& ABRAMIDES, E. Diferenças na oviposiçăo de Bemisia tabaci em variedades de soja. Anais da Sociedade Entomológica do Brasil, Jaboticabal 6(2):256-263, 1977.

TURNIPSEED, S.G. \& KOGAN, M. Soybean entomology. Annual Review of Entomology, 21:247-282, 1976. 